\title{
A Combined Batteryless Radio and WiFi Indoor Positioning for Hospital Nursing
}

\author{
Riad Kanan, and Obaidallah Elhassan
}

\begin{abstract}
This paper proposes a design of an efficient hospital nurse calling system which combines two types of indoor localization systems. The purpose of the first system is to locate patients while the second is to locate nurses equipped with their smart phones. The main goal of developing such system is to decrease the time taking for nurses to provide healthcare for patients. Patients' positioning system is RF based. Indeed, each patient is equipped with a wireless and battery-free call button. When the switch is pressed, a wireless telegram is sent to reference nodes that act like Wireless Sensor Networks (WSN). The positioning of patient is performed using trilateration method with the help of Received Signal Strength Indicator (RSSI) values. Hence, beacons will forward the received signal from patient's call button to a central receiver module connected to a computer. A dedicated program has been developed to calculate the position of the call button and post it on an online database. On the other hand, the nurses' localization system is WiFi-based. Nurses' positioning is done by determining the Time of Arrival (ToA) and the Angle of Arrival (AoA) between the mobile phone and the WiFi router. The mobile phone locations are posted to the online database as well. Our program performs a comparison between the nurses' and the patient's coordinates. The nearest nurse gets an alarm. As consequence, a patient gets care from the nearest available nurse in an efficient way and with less time. The proposed system is user-friendly and Internet of Things (IoT) based architecture integrating two heterogeneous localization systems seamlessly.
\end{abstract}

Index terms - Received Signal Strength Indicator (RSSI); Indoor Localization/Positioning; Location Awareness; EnOcean Standard; WiFi-Based Positioning System (WPS); Angle of Arrival (AoA); Time of Arrival (ToA); Trilateration; Internet of Things (IoT)

\section{INTRODUCTION}

With the progression and development of wireless sensor networks (WSN) and wireless technologies, a field of research that is strongly linked with it is location tracking. WSN nodes are getting smaller and more efficient, and data processing and network management became smarter [1]. Indoor positioning systems are made to locate and track objects or people by radio waves and signals in indoor environments. This is an alternative to GPS that is used for global positioning-outdoor [2]. Wireless based indoor localization technologies are many and classified in three main categories. Long distance positioning is

Manuscript received October 29, 2015; revised February 16, 2016. Authors are with Abu Dhabi University, Abu Dhabi, UAE. (E-mails: riad.kanan@adu.ac.ae,obaidallah.elhassan@gmail.com). done by FM (Frequency Modulation) or GSM/CDMA (cellular networks). Another category is for middle distances and based on $\mathrm{WiFi}$ or ZigBee for example. While Bluetooth, RFID, and UWB are for short distance localization. There are many techniques for indoor positioning, and they suit various needs and criteria of evaluation such as accuracy, coverage, infrastructure, update interval, computational cost, offline computing, and localization time [3] [4] [5]. Mainly, location systems for coordinate determination can be based on lateration, proximity, or angulation. From the technology point of view, classification of location systems can be categorized in a tree as shown in Fig. 1 [6] [7].

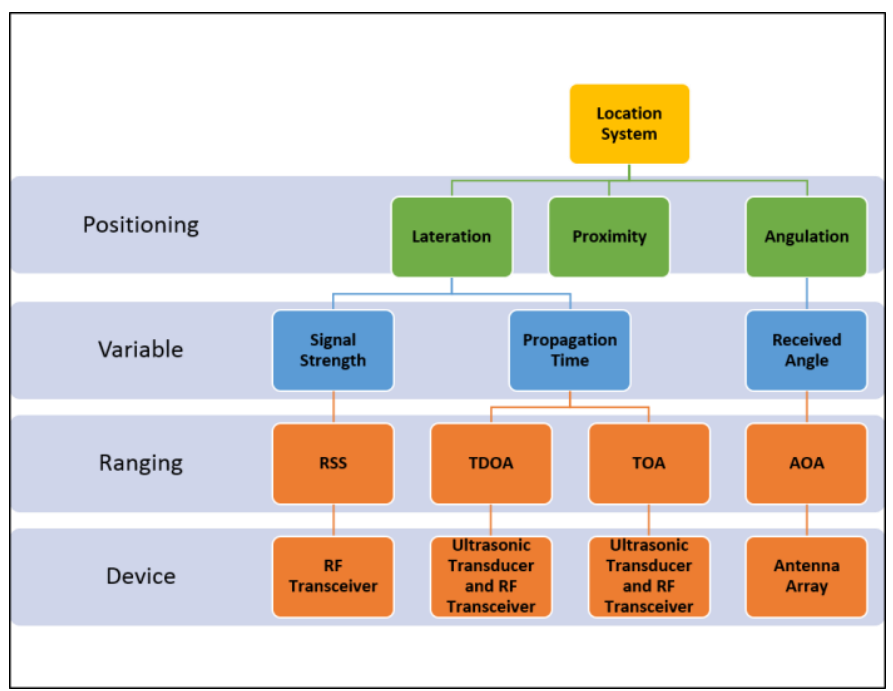

Fig. 1. Classification of Location Tracking Systems

There are many location-aware systems that are introduced over the years which use variety of indoor positioning techniques for many applications such as healthcare, homecare, monitoring, tracking, etc. [8]. For instance, there are Infrared (IR) positioning systems. These are the most common, cheap, and easy to install and maintain. The common applications are motion tracking and optical tracking cameras. The IR based system is considered to give accurate measures, yet the major disadvantage of this system is security and privacy. Another technique is the Ultrasound positioning system. Bats that use ultrasound waves for navigation inspired people to build a similar system. It is cheap, more secured, but less accurate because of reflected ultrasound signals and other kinds of noise such as jangling metal objects. Radio Frequency (RF) is strong 
so that it can travel through objects like walls with a larger covering area. Some existing infrastructure like WLAN APs can facilitate the operations of this system. RFID, WLAN, Bluetooth, Sensor Networks, and UWB are based on RF positioning. There are positioning systems that are based on magnetic signals. The advantages of this positioning system are that it offers high accuracy, does not suffer from line-of-sight problems, and offers multi-position tracking at the same time. Magnetic sensors are cheap, compact, and robust. However, the performance and accuracy are highly depending on the range of coverage. Therefore, there is a need to place higher number of sensor nodes in an area. Vision-based positioning system uses computer vision to track the locations and identify people or objects. The major advantage is that there is no need for any target nodes placed on objects nor held by persons. The challenges that face this system are visual effects like lights and weather. Also, it does not protect privacy nor reliable in dynamic environments, and it requires high computational abilities. Another type of positioning systems is the Audible Sound positioning system. Objects like mobile phones are tacked by acoustic sensors where the object emits an audible sound. It is low cost and considers privacy. On the other hand, it is noisy, does not have high penetration ability, and can be interfered by other sounds [8] [9].

Now indoor localization systems are strongly linked with the Internet of Things for a variety of applications in different fields. With the difference of IoT protocols and smart objects, heterogeneity represents a major challenge in building IoT based systems. To overcome this issue, L. Mainette et al proposed in [10] a user-friendly multi-protocol middleware platform that allows common individuals to develop services for home automation. Mainly, these services may take control over home appliances according to user's configurations and user's location [11].

To utilize our indoor positioning system, the radio standard considered for this application is EnOcean Standard. It is a new standard that has been published and recognized in 2012. EnOcean Radio Protocol is optimized and utilized for ultra-low power and batteryless technologies and applications. It is considered to be more reliable, wider in coverage, interoperable, and can co-exist with other radio systems. Unlike the universal $2.4 \mathrm{GHz}$ and sub $1 \mathrm{GHz}$ radio standards, EnOcean radio standard cover sub $1 \mathrm{GHz}$ bands e.g. ASK $868 \mathrm{MHz}$ according to R\&TTE regulation EN 300220, and it has a high data rate which is $125 \mathrm{Kbps}$. Also, it covers wide range, $300 \mathrm{~m}$ in free field and 30m in buildings [12] [13].

Based on EnOcean Radio Protocol, we designed and implemented a nurse calling system [14]. This system includes a patient call button which is battery-free and wireless as well. Furthermore, we developed a dedicated program to process incoming wireless telegrams transmitted by the call button and post them on an online database. On the nurse side, we designed a nurse station view to display patients' calls and a mobile application for nurses to receive notifications. In order to allow patients getting healthcare from the nearest available nurse, an improved version of the system has been developed [15]. In this new version, patients are equipped with a batteryless call button, and their locations are determined using trilateration method. Simultaneously, nurses hold mobile phones, and they are localized using a hybrid Time of Arrival (ToA)/Angle of
Arrival (AoA) method. Moreover, we validated the performance of the batteryless modules by conducting accuracy and precision tests which resulted in 3 meters accuracy. In this paper, we validated the performance of nurse positioning by conducting tests that consist of "ToA Performance Test", "AoA Performance Test", and "WPS Accuracy and Precision Test". These tests and simulations showed that the system achieved a performance of a great efficiency and accuracy.

This paper is organized as follows. Section II is presenting the system design. Section III shows the methodology implemented for the positioning of batteryless modules, and it shows localization accuracy test results, while, in Section IV, the implementation and performance validation of mobile phone localization via WiFi is explained. Section V shows the linking between both systems and the Internet of Things (IoT) behind it, and finally, Section VI concludes the paper.

\section{THE SYSTEM AND TOPOLOGY DESIGN}

The patient call button as shown in Fig. 2 is powered by EnOcean Radio Standard. It uses mechanical energy harvesting where a small mechanical motion (Fig. 3 [12]) is converted into an electrical energy that is enough to power up the RF transmitter.

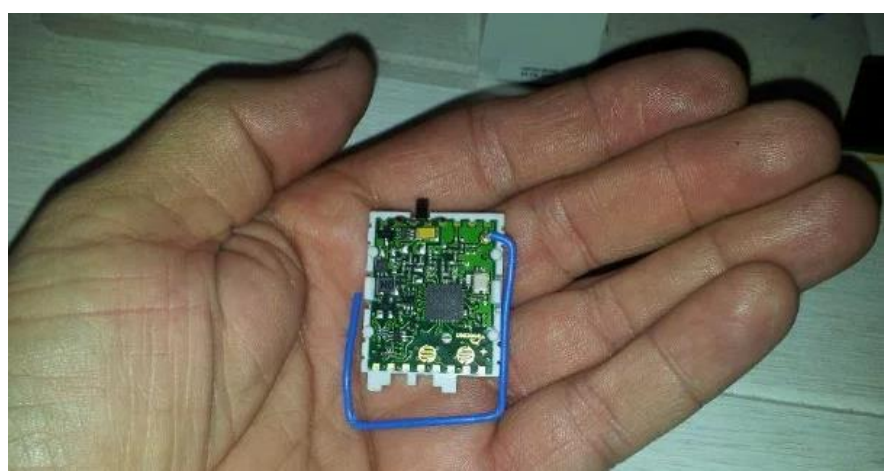

Fig. 2. Handheld Batteryless Wireless Pushbutton Transmitter Device

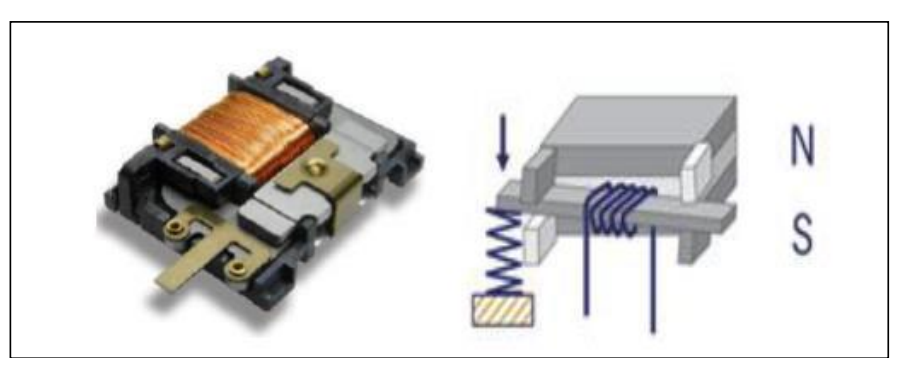

Fig. 3. Energy Harvester

The $7 \mathrm{~N}$ human push on the generator generates $0.5 \mathrm{mWs}$ in average [12] [13] [16], and this pulse has a magnitude that varies between $7 \mathrm{~V}$ and $15 \mathrm{~V}$ with a pulse width of $2 \mathrm{~ms}$ (Fig. 4). This is when no load is connected. When the harvester is loaded by the transmitter circuit, the energy demand is limited between $120 \mathrm{uWs}$ and $210 \mathrm{uWs}$. Thus, the generator can supply $200 \%$ more energy than required. 


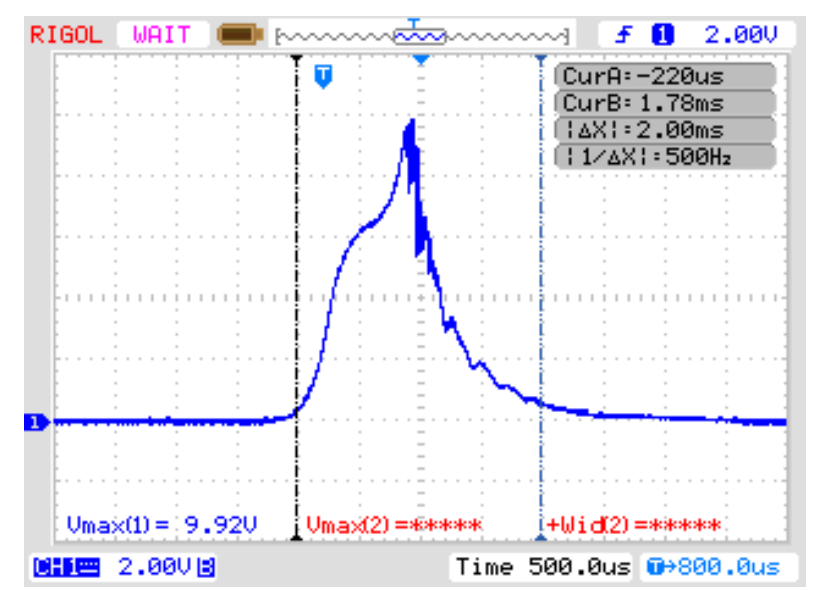

Fig. 4. Energy Harvester Pulse

The voltage supplied by the harvester is an AC pulse which can be between $4 \mathrm{~V}$ and $7 \mathrm{~V}$ with a time of $4 \mathrm{~ms}$. This pulse can be either positive or negative based on the polarity. The power conditioning circuit is made to provide the desired power level for the microprocessor. The stages of the conditioning circuit are as the following:

- AC/DC Converter: Electronic converter unit for generating the device DC power supply.

- Storage Capacitor: $10 \mu \mathrm{F}$ that stores the harvested energy and smoothens the DC voltage to constant voltage for maximum $50 \mathrm{~ms}$.

- DC/DC Converter: Electronic converter unit for reducing the voltage to the desired level which should not be more than $3.3 \mathrm{~V}$ as in Fig. 5 .

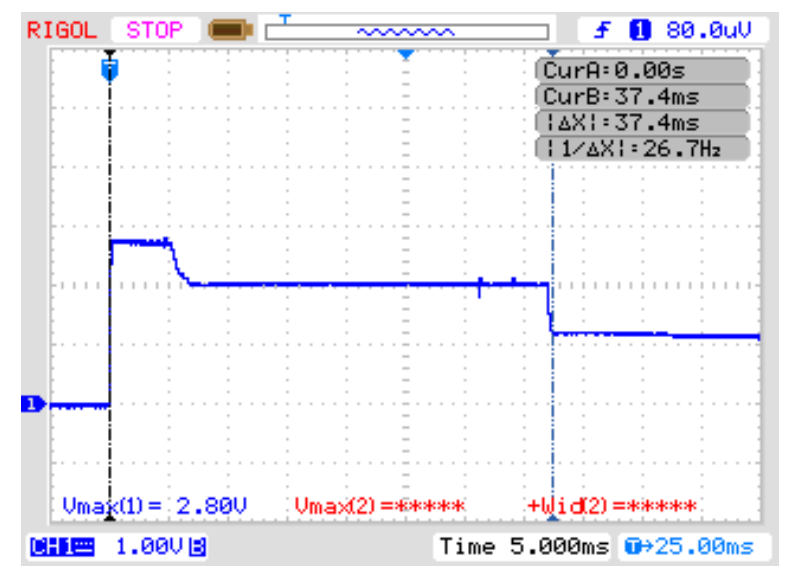

Fig. 5. Step-down Converter Output

When an energy pulse is supplied, an RF telegram is transmitted including a unique 32-bit module ID as an RPS Telegram (Fig. 6). It is a type of telegrams for Repeated Switch Communication, and it gives 1 byte content data [17]. The RPS telegram content can be configured if other content is needed. The setup of the transmission timing allows avoiding possible collisions with data packages of other EnOcean transmitters as well as disturbances from the environment.

\begin{tabular}{|c|c|c|c|c|}
\hline 1 & 1 & 4 & 1 & 1 \\
\hline RORG & DATA & TXID & STATUS & HASH \\
\hline
\end{tabular}

Fig. 6. RPS Telegram Structure

With each transmission cycle, 3 identical sub telegrams are transmitted within $40 \mathrm{~ms}$. The transmission of a sub telegram lasts approximately $0.7 \mathrm{~ms}$. The delay between the three transmission bursts is affected at random within that range which is $40 \mathrm{~ms}$ (Fig. 7, 8) [18].

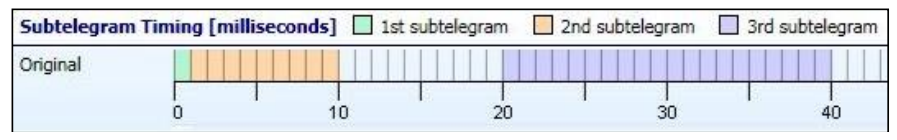

Fig. 7. Telegram Timing

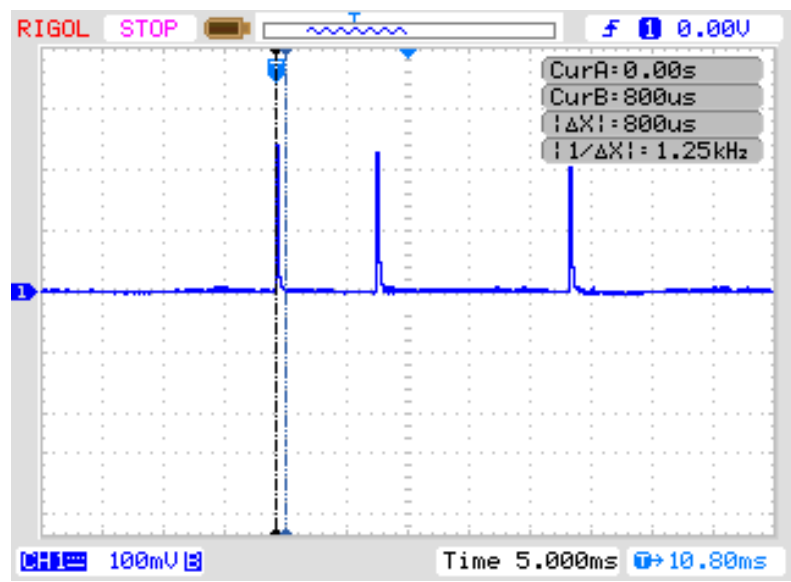

Fig. 8. RF Output

The transmitted signal from the switch is a 21 byte data word, and when it is received, many data can be extracted like source ID, destination ID, RSSI value, and other information that is meaningful to the user [19]. To test the technology, we had one transmitter and a receiver attached to PC. The telegram is viewed an analyzed using a computer software as in Fig. 9. The ID column represents the module ID, and RORG is telegram type. Data and Status columns are interpreted depending on the application set by the user. $d B m$ is the RSSI value. Subtel is number of the strongest sub telegram received since there a sum of three sub telegrams. DestinationID can be set by the user, so the telegram reaches a specific destination; "FFFFFFFF" destination ID means that the telegram is broadcasted [17].

\begin{tabular}{|c|c|c|c|c|c|c|c|}
\hline ID $\nabla$ & RORG 7 & 7 & Data & Status? & $\mathrm{dBm}$ & Subtel & DestinationID \\
\hline $002 A 1 A C 7$ & RPS & 50 & & BO & -41 & 1 & FFFFFFFF \\
\hline $0188 F 6 D 5$ & VLD & $4 \mathrm{~B}$ & & 86 & -73 & 1 & 002A1AC7 \\
\hline $018826 \mathrm{BB}$ & VLD & $4 \mathrm{~B}$ & & 84 & -49 & 2 & 002A1AC7 \\
\hline $002 A 1 A C 7$ & RPS & 00 & & $\mathrm{AO}$ & -41 & 1 & FFFFFFFF \\
\hline $0188 F 6 D 5$ & VLD & $4 \mathrm{~B}$ & & 86 & -72 & 1 & 002A1AC7 \\
\hline $018826 \mathrm{BB}$ & VLD & 46 & & 84 & -51 & 1 & $002 A 1 A C 7$ \\
\hline
\end{tabular}

Fig. 9. Received Telegrams

The RSSI value over distance in an indoor line-of-sight is tested, and the results are shown in Fig. 10. According to the 


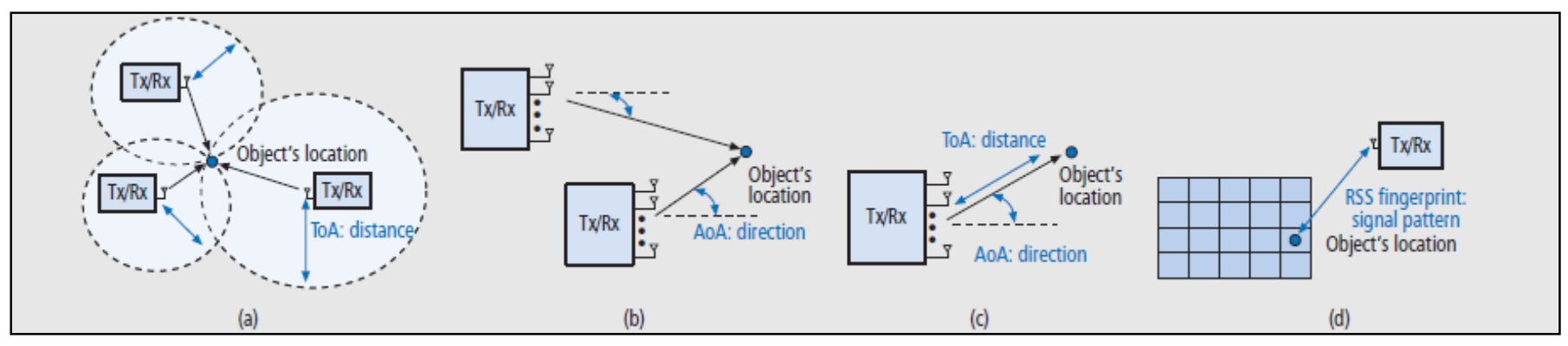

Fig. 11. a) Time of Arrival Approach; b) Angle of Arrival Approach; c) Hybrid ToA/AoA Approach; d) Received Signal Strength and Fingerprint Approach

figure, many samples are taken at one position, and there is between $7 \mathrm{dBm}$ and $10 \mathrm{dBm}$ deviation at a position. For the transmitter module, RSSI values are between $-40 \mathrm{dBm}$ for good signals and $-90 \mathrm{dBm}$ for weak signals [18].

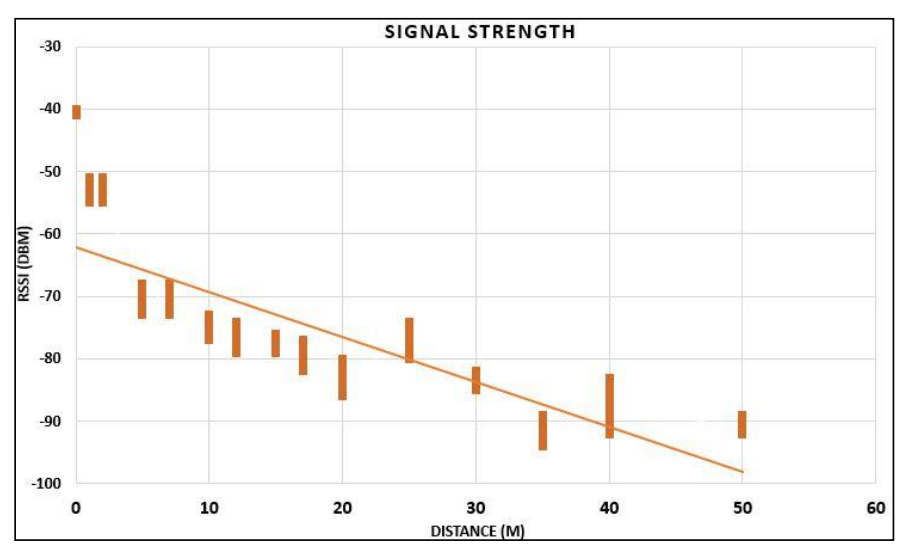

Fig. 10. RSSI vs. Distance

Many factors affect the RSSI value [20]. Perhaps the most factor that should be put into consideration is the Path Loss Exponent (PLE). [21] Defines it as "all of the lossy effects associated with distance, and the interaction of the propagating wave with the objects in the environment between the antennas". Table I [22] shows variation of path loss according to the medium of radio wave propagation. As this system is implemented in hospital, the environment classification would be Obstructed in-building, so $4<\mathrm{n}<6$.

TABLE I

PAth Loss Exponent (PLE) With ResPeCt to ENVIRONMENT

\begin{tabular}{|l|c|}
\hline Environment & Propagation Exponent (n) \\
\hline Free space & 2 \\
\hline Urban area & $2.7-3.5$ \\
\hline Shadowed urban area & $3-5$ \\
\hline In building line of sight & $1.6-1.8$ \\
\hline Obstructed in-building & $4-6$ \\
\hline Obstructed in-factories & $2-3$ \\
\hline
\end{tabular}

To determine the position of the call button, needed is a network of beacons that receive the transmitted telegram and process the signal to generate coordinate. Among many methods and estimations, the considered method is trilateration where three beacons are already located at known positions
[23]. Upon call, the button's distance is measured with respect to RSSI value, and the position is determined [24].

$\mathrm{WiFi}$ is widely applied in indoor environments. With the already existing infrastructure like Access Points (AP) and mobile phones, we make use of this advantage and propose the WiFi-Based Indoor Positioning System. There are different methods to locate WiFi devices as illustrated in Fig. 11 [25]. There is Time of Arrival (ToA), Angle of Arrival (AoA), hybrid (AoA/ToA) which we will implement, and RSS footprint. Each one these approaches has its advantages and limitations [25] [26] [27].

Time of Arrival (ToA) is the travel time between a transmitter and a receiver, and by multiplying it with the speed of light $\left(3 \times 10^{8} \mathrm{~m} / \mathrm{s}\right)$, the distance can be calculated. This approach requires the transmitter and the receiver to be synchronized. Also, for 2D localization, at least three $\mathrm{WiFi}$ routers are required while four routers are needed for $3 \mathrm{D}$ localization. The performance and the accuracy of ToA approach is constrained by the signal's bandwidth and its sampling rate. For example, a $10 \mathrm{MHz}$ signal has a resolution of $10 \mu \mathrm{s}$ in time domain, so the resulting positioning error is $3 \times 10^{8}$ $\times 10 \times 10^{-6}=30 \mathrm{~m}$. If the bandwidth is increased to $1 \mathrm{GHz}$, the error is distance is down to $30 \mathrm{~cm}$. Therefore, the higher the bandwidth, the less the error. UWB solves this issue with an accuracy of $1 \mathrm{~cm}$, but this technology requires very high bandwidth, special hardware design, and very high cost [25].

Angle of Arrival (AoA) method determines the incoming signal's direction from the transmitter on the antenna array. The direction is calculated via exploitation and detection of phase difference among antennas. For that, two WiFi routers with antenna arrays are required. However, the positioning accuracy for line-of-sight falls significantly due to multi-path effects where the signals bounces of objects ahead such as walls, furniture, and human beings. A commercial product named as "Quuppai HAIP" solves the problem with an accuracy of $0.5 \mathrm{~m}$ to $1 \mathrm{~m}$, yet a specialized device with 16 array antennas with transmitters and Bluetooth for signal enhancement, tagging, and overcoming multi-path effect are needed [25].

The hybrid (ToA/AoA) WiFi-Based positioning technique can improve the localization performance from the bottleneck. Unlike the traditional approaches, this mechanism relaxes the need for wide signal bandwidth and large numbers of antennas and routers by utilizing the transmission of multiple predefined messages while maintaining high-accuracy performance. Also, it helps with addressing the issue of complexity of environment and limited number of routers. Now, a single multi-antenna WiFi Access Point (AP) is enough to locate objects. Still, the 
hybrid approach suffers high bandwidth and number of antennas challenges, but it leverages the benefit from both ToA and AoA approaches [25].

\section{A. Patient's Call Button}

To determine the distance from signal strength, Eq. (1) is adopted. $D$ is distance between Tx and Rx in meters. RSSI is the reference signal strength at $1 \mathrm{~m}$ in $\mathrm{dBm}$ which is $-41 \mathrm{dBm}$ according to our "RSSI vs. Distance" test results. $A$ is the RSSI value at any distance, and $n$ is Path Loss Exponent (Table I) [20].

$$
D=10^{\frac{R S S I-A}{10 n}}
$$

The positioning technique used in the design is Trilateration Estimation. It is used to find the unknown position of the target node inside a WSN of reference nodes. There will be three reference nodes and one target node [6] [28]. When trying to locate the target node, there is a wireless communication between target node and each reference node (beacon). Resulting from this communication is a distance. Three radii and the target node illustrate an intersection point. By using Pythagorean Theorem in Eq. (2), the coordinates of the target node or the switch can be determined.

$$
\begin{aligned}
& d_{1}^{2}=\left(x_{1}-x\right)^{2}+\left(y_{1}-y\right)^{2} \\
& d_{2}^{2}=\left(x_{2}-x\right)^{2}+\left(y_{2}-y\right)^{2} \\
& d_{3}^{2}=\left(x_{3}-x\right)^{2}+\left(y_{3}-y\right)^{2}
\end{aligned}
$$

\section{B. Nurse's Cell Phone}

To measure the distance without requiring time synchronization between stations - mobile phone and access point, we apply Round-Trip Time (RTT) method. The receiver gets transmitted signals and records the timestamp as $t_{S}$. When the receiver acknowledges a receipt of the message, RTT can be calculated from the interval between sending and arrival times. The response time started in Eq. (3) is measured and allocated by the ToA approach. The $T_{U}$ is the agreement time between transmitter and receiver, and $i$ is the arbitrary number decided by the receiver for allocation convenience. Once the transmitter estimates the first message by getting arrival time's stamp $t_{R}$, the RTT is shown in Eq. (4) [25].

$$
\begin{gathered}
t_{s}^{\prime}+i \times T_{U} \\
R T T=\left(t_{R}-t_{S}\right) \bmod T_{U}
\end{gathered}
$$

Regarding Angle of Arrival (AoA), cell phone will transmit number of signals, so that it will get measured using estimation channel. Two antennas are enough to obtain AoA, and the more the antennas, the better the estimation [25] [29].

\section{BATTERYLESS MODULES LOCALIZATION SYSTEM}

Based on the discussion in Section II A, the approach followed to locate target nodes is through RSSI, and the estimation technique is the Trilateration Estimation. Three beacons are needed, and, therefore, three distances will result with an intersection point. After that, by applying Eq. (1), $(x, y)$ will be obtained.

To achieve that, three reference nodes are triangularly placed in known locations, and each of them have a unique module ID. The message transmitted by the beacon to the receiver terminal is modified as it contains the module ID of the target node, the received signal strength between target node and reference node, and the reference node's module ID. The USB receiver converts RF message into serial data, and the data can be viewed and processed. We have written a code so we get these information. After signal gets received, distance is calculated using Eq. (1), and the position is obtained via Eq. $(5,6) . u$ is distance between first and second beacon while $v$ is the distance between first and third beacon (Fig. 12).

$$
\begin{aligned}
& x=\frac{u^{2}+\left(d_{1}^{2}-d_{2}^{2}\right)}{2 u} \\
& y=\frac{v^{2}+\left(d_{1}^{2}-d_{3}^{2}\right)}{2 v}
\end{aligned}
$$

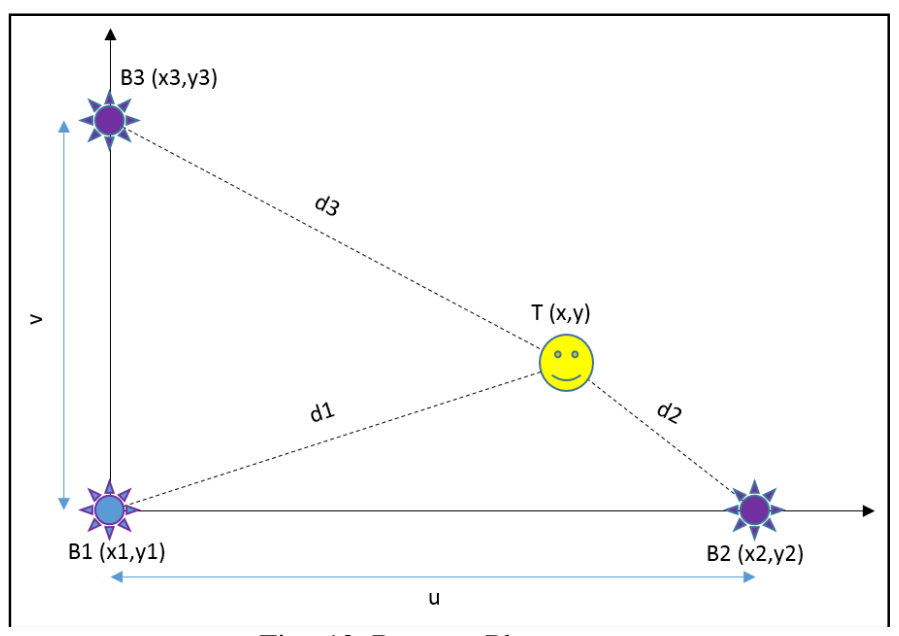

Fig. 12. Beacons Placements

\section{A. Accuracy and Precision Test for Batteryless Radio Localization}

Positioning precision and accuracy test is implemented and conducted as follows. There are three pre-determined positions for the switch. At each position, the patient switch is triggered five times. The test results are shown in Fig. 13 and show that the position can be accurate up to 5 meters ( 3 meters in average). Since we are not tracking small objects but humans, this margin of error is acceptable. Table II shows the real coordinates. 


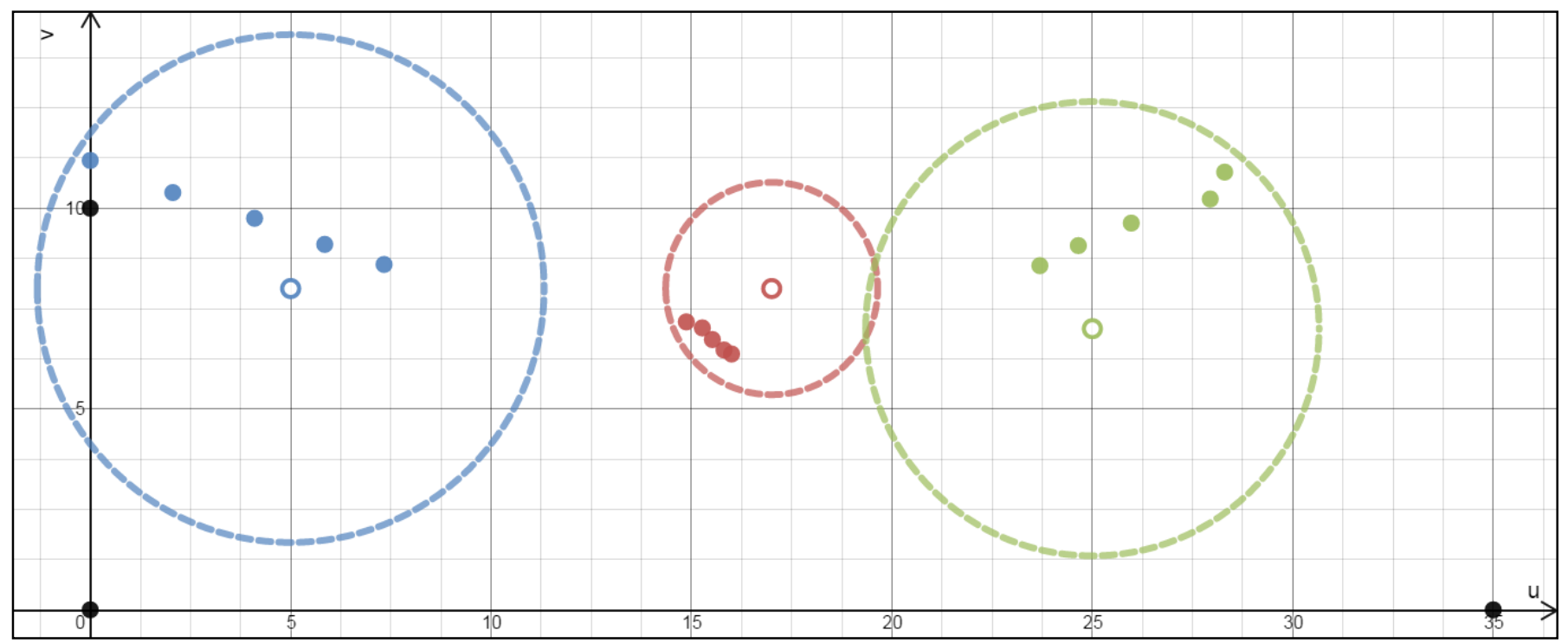

Fig. 13. Batteryless Modules Positioning Accuracy and Precision Test

TABLE II

TARGET AND REFERENCE NODE’s POSITIONS

\begin{tabular}{|l|c|}
\hline Node & $(\mathbf{x}, \mathbf{y})$ \\
\hline Beacon 1 & $(0,0)$ \\
\hline Beacon 2 & $(35,0)$ \\
\hline Beacon 3 & $(0,1)$ \\
\hline Target Node 1 (Blue) & $(5,8)$ \\
\hline Target Node 2 (Red) & $(17,8)$ \\
\hline Target Node 3 (Green) & $(25,7)$ \\
\hline
\end{tabular}

\section{WIFI-BASED Positioning SYSTEM}

Section II B discussed the methodology to find the distance between WiFi AP and cell phone. This section tells the positioning procedure. The mobile phone begins requesting service from the AP for positioning. Then the AP will reply back to the mobile with RTT measurement requests. This is done by sending multiple messages from the AP to mobile and recording timestamp. The data word of each message is the same, but the message contain different sub frequencies of OFDM symbol [25] [30] [31].

The mobile processes the RTT requests, measures arrival time, and sends multiple RTT results to the AP with respect to the time difference by the already decided value of arbitrary factor $i$ as in Eq. (3) to send the burst $M$ messages where each message contains multiple sub frequency pilots [25].

RTT and AoA are measured by the AP in terms of user device (mobile phone). Distance is calculated as in Eq. (7).

$$
D=\frac{R T T \times 3 \times 10^{8}}{2}
$$

The AoA is measured by using the message nearest the ToA to estimate channel state information. The AP delivers its location, its ID, and the distance between AP and mobile device. Finally, Mobile phone uses ToA/AoA to locate its own position [25] [32].

\section{A. ToA/AoA Performance Tests}

Simulations are done to investigate the ToA/AoA positioning performance in terms of accuracy and precision. The sampling rate and bandwidth are assumed to be between $20 \mathrm{MHz}$ and 40 MHz. Also, FTT sized are assumed to be from 64 to 128. Every AP has four antennas while mobile phone is equipped with one antenna. The time among burst messages is $T_{S} / M$, for $M$ is the number of messages, which is between 10 and 20 messages and 16 to 32 pilot assistance [25].

Fig. 14 shows distance in meters between the router and the mobile device versus the Signal to Noise Ratio (SNR) in decibels. It can be referred from the simulation result is that 20 messages in $20 \mathrm{MHz}$ bandwidth is similar to 10 messages for a bandwidth of 40MHz. This means a higher precision [25].

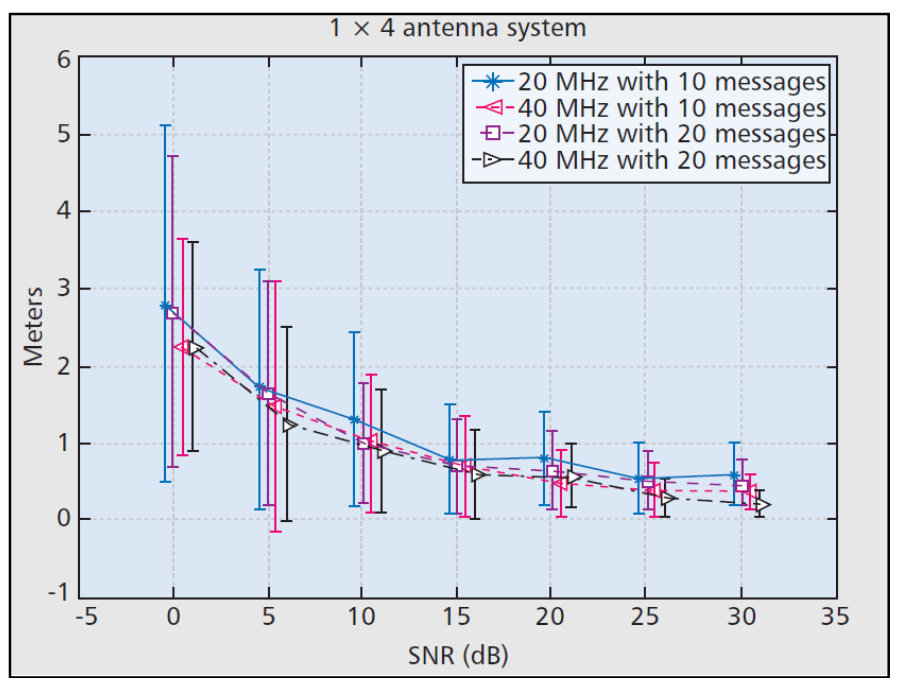

Fig. 14. ToA Approach Performance

For the Angle of Arrival (AoA), the performance of phase against SNR is shown in Fig. 15. The performance can get more 
efficient by including more burst messages with larger bandwidth for higher SNR. However, the performance for the $40 \mathrm{MHz}$ is lower than performance for $20 \mathrm{MHz}$ when SNR is low because FFT size of 127 is lengthy, and its sensitivity of channel estimation is very high. Therefore, for high SNR, AoA for the message with 32 pilots is much better than message with 16 pilots [25]. The localization error from AoA depends on the distance between the $\mathrm{WiFi}$ router and the mobile phone since the measurement error of angle multiplied by the distance is a localization error. Considered is a scenario where the SNR is $20 \mathrm{~dB}$ and a WiFi AP's maximum indoor distance is $50 \mathrm{~m}$. By utilizing 10 burst messages, a single WiFi AP can achieve $2.2 \mathrm{~m}$ and $1 \mathrm{~m}$ accuracy for $20 \mathrm{MHz}$ and $40 \mathrm{MHz}$ bandwidth, respectively.

When two or more Access Points are deployed, the position performance can reach $2.2 \mathrm{~m}$ and $0.5 \mathrm{~m}$, respectively. When SNR is low, the noise factor plays an important role to affect phase measurement. The time shift property that refers to the time difference between messages is not precise, and the performance is downgraded. Nevertheless, this mechanism gives superior performance under current WiFi AP hardware conditions [25].

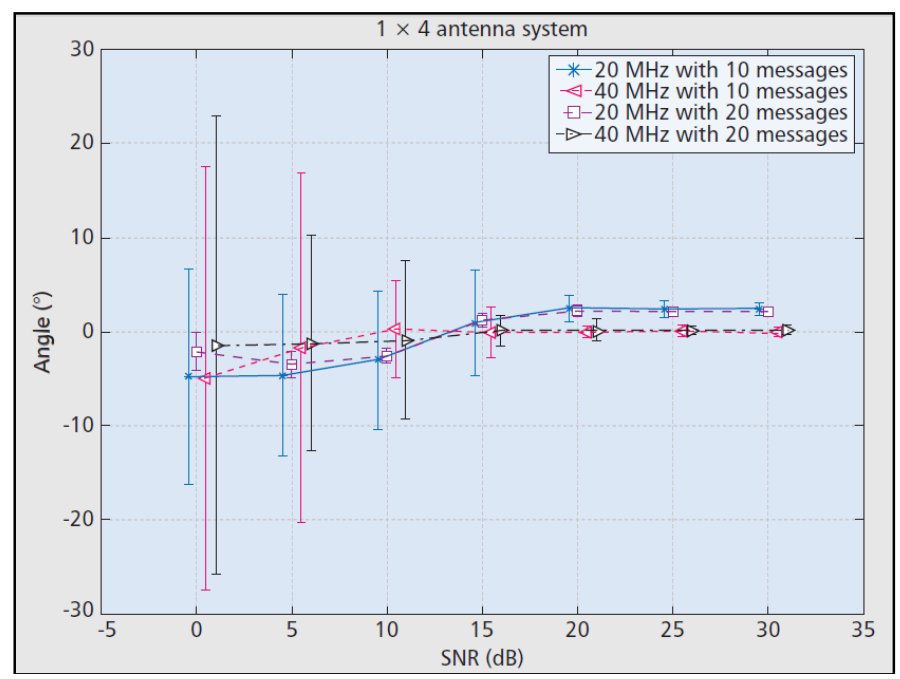

Fig. 15. AoA Approach Performance

\section{B. Accuracy and Precision Test for WiFi Localization}

Another Positioning precision and accuracy test is implemented for the WiFi-based system. In this test, a WiFi Access Point is placed on a fixed position, and a WiFi device is mobilized to three different positions. At each position, 10 Internet Control Message Protocol (ICMP) pings are sent to the Access Point and 10 responses in return. The round trip time (RTT) is measured in the order of milliseconds. This resulting RTT has combined the various types of network delays, which are processing delay, queuing delay, transmission delay, propagation delay, etc. Our interest is in obtaining the RTT of propagation. However, these delays tend to be constant, so they are treated as offset for elimination. The propagation delay has a linear rate of change with respect to distance between nodes [33]. For indoor environments, the target RTT should be in the order of sub-microseconds. Continuing on the same scenario of the simulation, the test results are shown on Fig. 16 while further details are provided in Table III.

\section{TABLE III}

WIFI ROUTER AND MOBILE POSITIONS

\begin{tabular}{|l|c|}
\hline Node & (Distance, Angle) \\
\hline WiFi Access Point & $\left(0,0^{\circ}\right)$ \\
\hline Mobile Position 1 (Blue) & $\left(5,315^{\circ}\right)$ \\
\hline Mobile Position 2 (Red) & $\left(10,45^{\circ}\right)$ \\
\hline Mobile Position 3 (Green) & $\left(15,180^{\circ}\right)$ \\
\hline
\end{tabular}

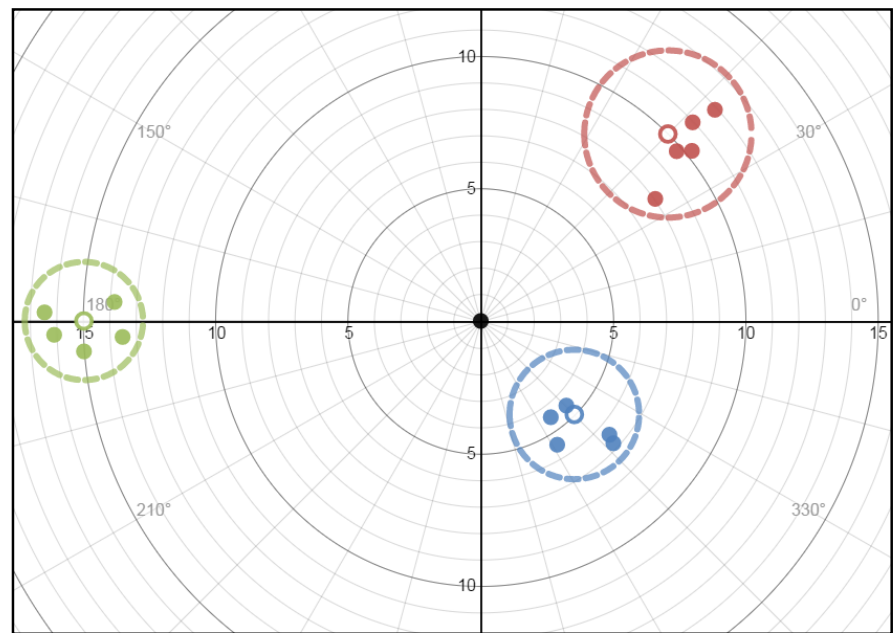

Fig. 16. WiFi-Based Positioning Accuracy and Precision Test

\section{SYSTEMS INTEGRATION AND INFORMATION TECHNOLOGY}

This section reviews our actual nurse calling system named as "Batteryless Radio System for Hospital Application" and proposes a schema for integrating previously discussed localization systems which are the batteryless modules indoor localization and WiFi-based indoor localization. The section also expresses the IoT behind the system, and how the different components are seamlessly integrated.

\section{A. The Current Nurse Calling System}

The working principle of our current system is that, upon a switch click, a data word is wirelessly transmitted. There is a whole system and network to make an integration of this data word into a meaningful alarm message. In Fig. 7, there is a full interaction between the system components. Upon a patient call request, the signal is transmitted. The receiver filters and routes the call to the server. The server is an online database, so the nurse station and the nurse pager gets the alarm as in Fig. 18 [14].

The receiver is a small USB stick that is equipped with the TCM 310 transceiver module. It provides bidirectional EnOcean radio protocol to devices with USB interface Radio messages are sent and received through a virtual serial interface (FTDI Virtual COM Port). It provides additional information like signal strength (RSSI) [34]. A code is built to receive the RPS telegram and extract an 8 byte unique tag ID. This ID is 


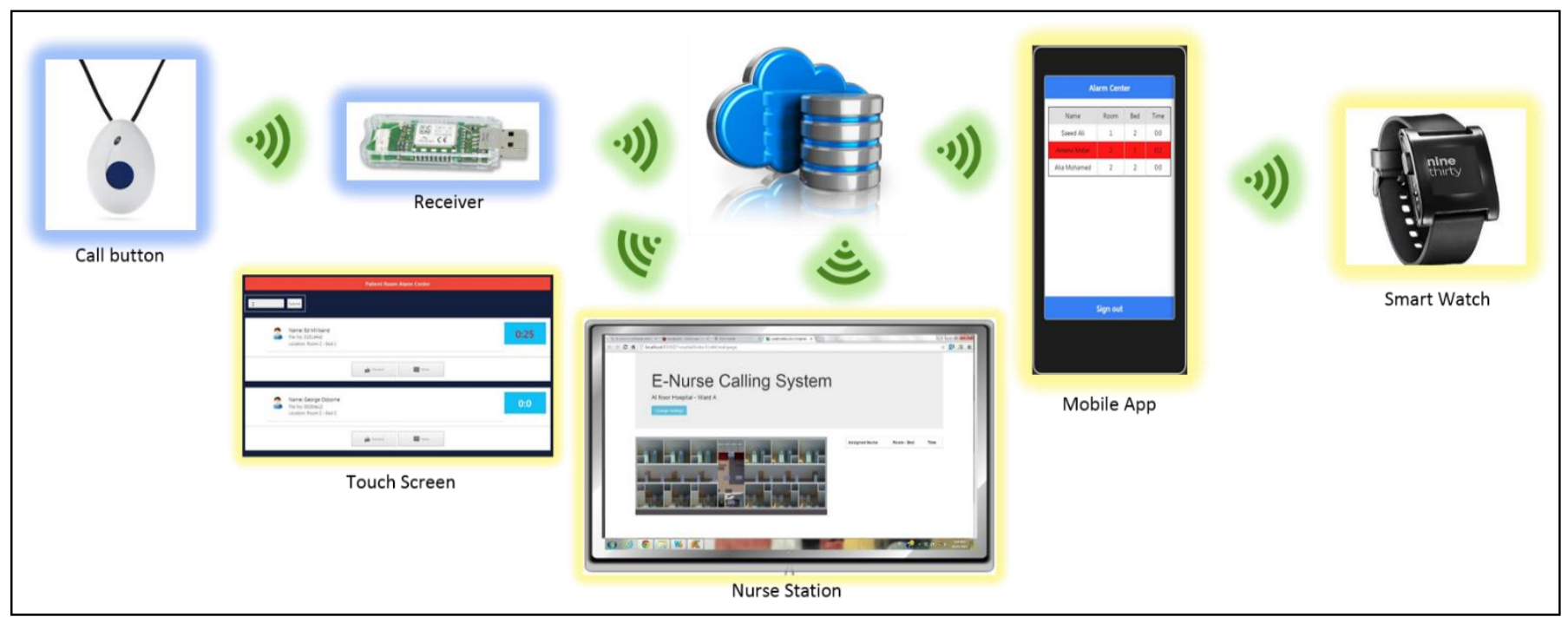

Fig. 17. Current System Architecture

uploaded and posted on a JSON based database server. Our database is based on JSON and uploaded on a web server. Patients' information inputs are uploaded. When a button is pressed, the status is updated in the server, and alarms are activated. For the nurse station, a map of each ward which can be uploaded to the server. The information of the patient can be also viewed or edited from the nurse station. When the switch is pressed, an alarm will be activated with a red spot on the patient's location and can be viewed in the server. A timer will start to show the time from the switch is pressed the alarm can be turned off from the nurse station [14].

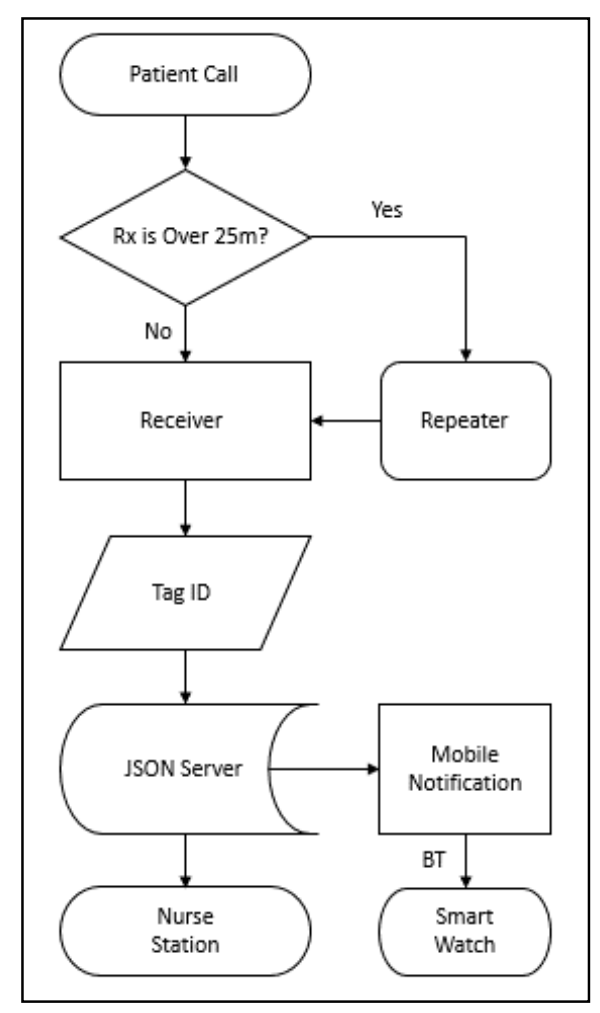

Fig. 18. Flow of Data for the Current System
The mobile application is dedicated for each nurse since each nurse is assigned to a number of patients. The patients that the nurse is assigned to are in the nurses' accounts. The mobile applications allow the nurse to sign in with a private username and password. Once signed in, a list of the patients will appear with a call status and location. Should the patient need help, a red blinking alarm, and local notification will appear in parallel with the central nurse station alarm system. Receiving notification has advantages over traditional systems. No need for noisy alarms that are placed all over the ward, and the nurse reaction will be faster since immediate recipient of the alarm. Patients' rooms are provided with a touch screen. This touch screen or tablet PC show the status of each patient in a particular room. The nurse can acknowledge that a patient has been checked and turn off the alarm using the touch display. It shows a timer, and notes can be added on patient's file as well [14].

An extra smart feature to the system is the smart watch. The smart watch is wearable by the nurse and paired via Bluetooth with the mobile phone. Once an alarm reaches the mobile phone, it gets forwarded to the smart watch. The watch is low power, for its battery life is up to 1 week [35]. Moreover, we are using the repeaters to extend the range for the communication between the switch and the nurse station. In the repeater, we are using TCM320 chip. The typical range for transmission inside hospital environment is approximately $25 \mathrm{~m}$ to $30 \mathrm{~m}$ and $50 \mathrm{~m}$ in corridors [36]. The repeater installation is based on the RSSI value. RSSI is received signal strength indicator. For the transmitter module, RSSI values are between $-40 \mathrm{dBm}$ for good signals and $-90 \mathrm{dBm}$ for weak signals [18]. Therefore, a safety margin of $25 \mathrm{dBm}$ is considered while placing a range extender which is $-65 \mathrm{dBm}$ as we consider $90 \mathrm{dBm}$ is our extreme end [14].

\section{B. The Proposed System}

The positions obtained from the batteryless modules localization system and the WiFi-based localization system will be posted to our existing online database which is hosted on a JSON based server. Fig. 19 shows the flow of information 


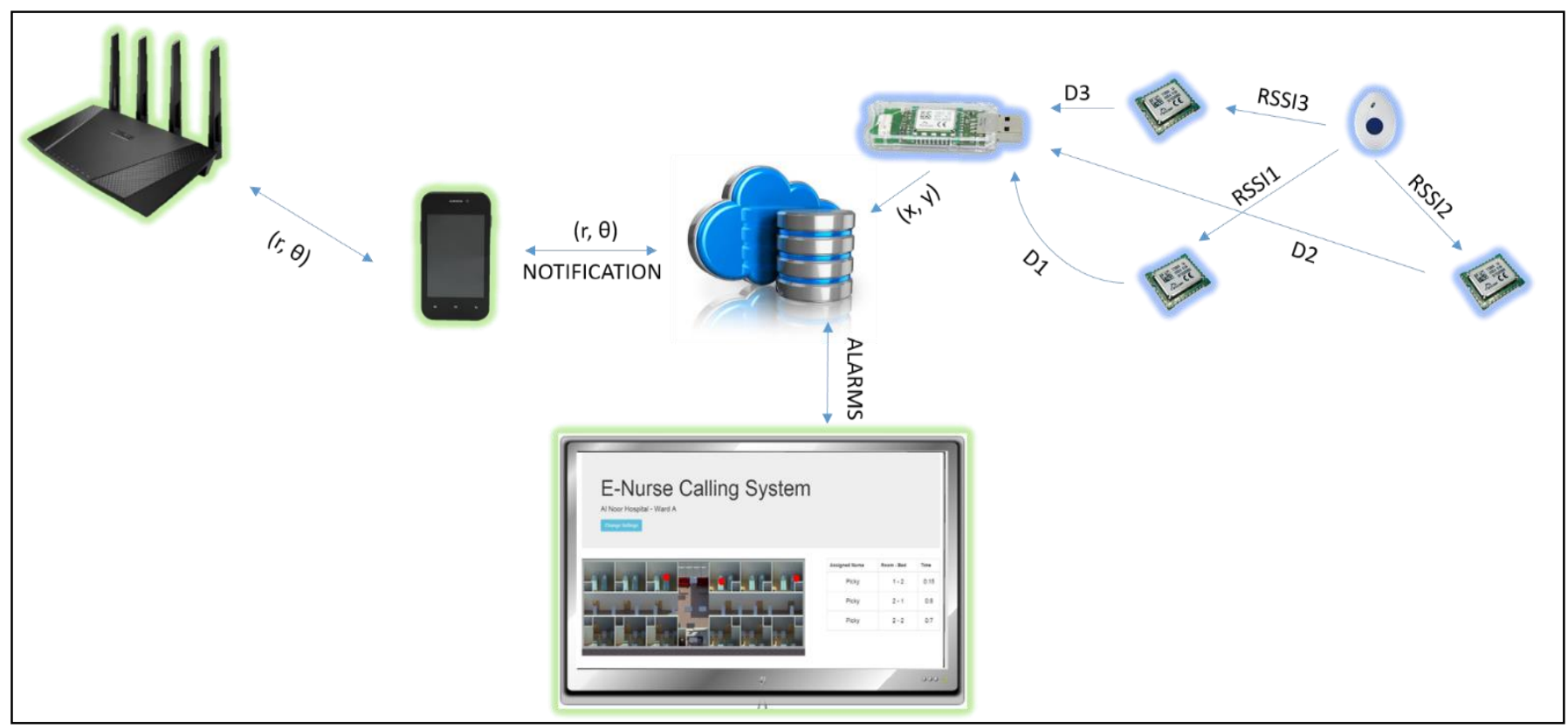

Fig. 20. Proposed System Architecture

through and the linking between the systems' components. The localization and notification processes are as follows:

1. Upon patient call, beacons that received the signal forward it to the receiver.

2. The strongest three RSSI readings between target node and reference nodes are considered.

3. Via trilateration method, the coordinates are determined.

4. The WiFi-based positions and phone owner information data are retrieved from the online database and compared to the determined coordinates of the patient.

5. The assigned nurse and patient coordinates are posted to the online database.

6. The patient location will appear on the nurse station view along with nearest nurse's name.

7. The call will be forwarded to the nearest nurse's mobile phone.

Fig. 20 shows an overview for the proposed system. The nurse station view is located on each ward. It has two main components which are a floor plan and alarms table. In the floor plan, the patient's location is shown upon a call. The alarms table tells the patient's name, location, elapsed time, and nearest nurse. The location is given in terms of a predefined zones such as TV Room, Room 3, WC, and so on. Therefore, the free nurse that avails in patient's zone or in the nearest zone will get a notification.

Regarding the mobile application, the main view is brief information of calling patient such as patient's photo, file number, age, zone, elapsed time since call. There is a button for the nurse to tab on to indicate that the needed care has been given.

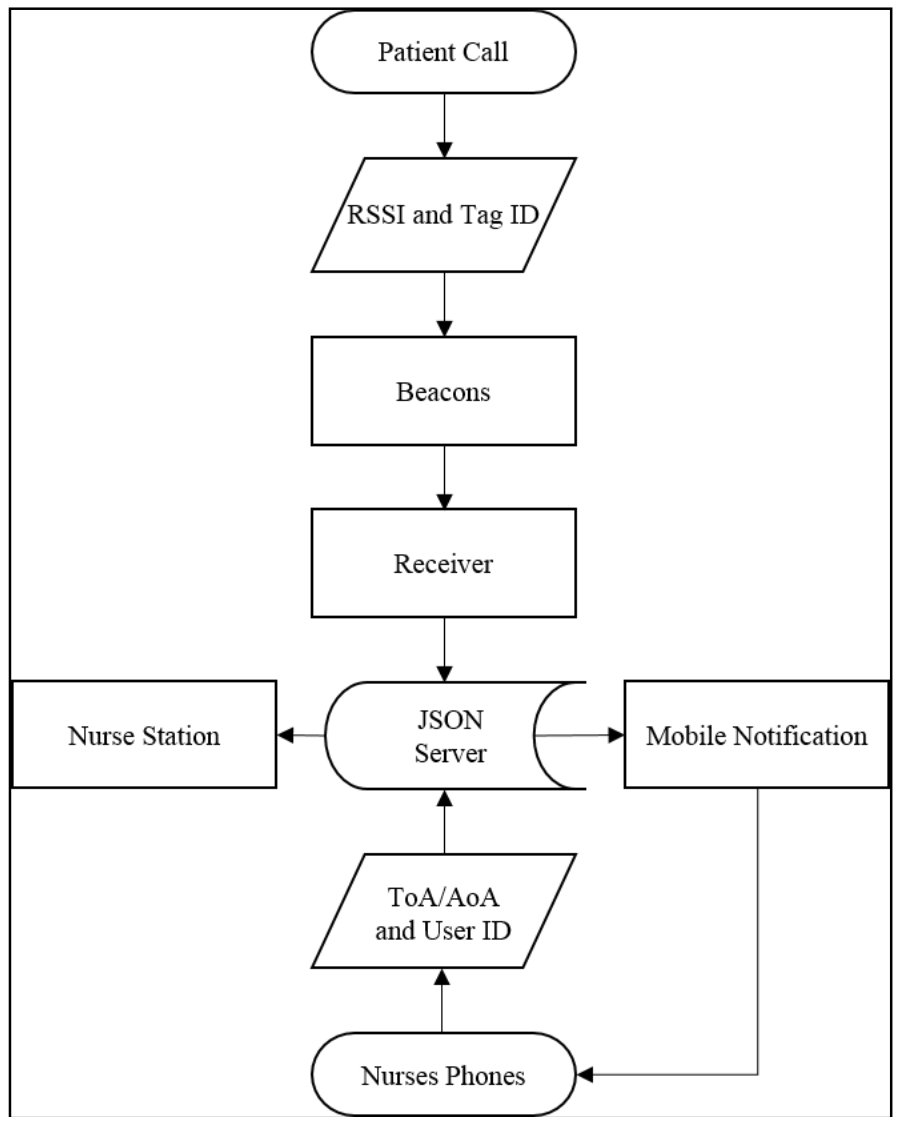

Fig. 19. Flow of Data for the Proposed System 


\section{CONCLUSION}

In this paper, we have presented a development and experimental validation for an effective batteryless nurse calling system that is able to locate patients and nurses in hospitals. The call button held by the patient is very small, batteryless, and wireless. Patient is tracked by distributed RF nodes in the hospital environment. The used technique is signal strength, and position is determined via trilateration. Simultaneously, the nurse holds a smart phone and gets located using WLAN access points; by time of arrival and angle of arrival, the nurse is spotted.

The key aim of the designed system is to increase efficiency of care giving to patients. Therefore, time to reach the patient will decrease considerably and, hence, providing higher quality health services. Also, a great feature of this batteryless system that it is a self-powered wireless transmitter and maintenancefree. The system installation requires no effort where the beacons are standalone, and the call buttons are wireless; additionally, the system makes use of the already existing WLAN infrastructure. Moreover, the proposed system satisfies the application requirements by achieving 3 meters accuracy for batteryless modules and 2 meters for mobile phones.

\section{REFERENCES}

[1] K. K. Chintalapudi, A. P. Iyer, and V. Padmanabhan, "Indoor localization without the pain," in Mobicom. Association for Computing Machinery, Inc., September 2010. [Online]. Available:

http://research.microsoft.com/apps/pubs/default.aspx?id=135 721.

[2] D. Petrovic and R. Kanan, "Extremely low power indoor localisation system," in Mobile Adhoc and Sensor Systems (MASS), 2011 IEEE 8th International Conference on, Oct 2011, pp. 801-806.

[3] J. Liu. (2014) Survey of wireless based indoor localization technologies. [Online]. Available: http://www.cse.wustl.edu/ jain/cse574-14/ftp/indoor.pdf

[4] D. Lymberopoulos, J. Liu, X. Yang, R. R. Choudhury, V. Handziski, S. Sen, F. Lemic, J. Buesch, Z. Jiang, H. Zou, H. Jiang, C. Zhang, A. Ashok, C. Xu, P. Lazik, N. Rajagopal, A. Rowe, A. Ghose, N. Ahmed, Z. Xiao, H. Wen, T. E. Abrudan, A. Markham, T. Schmid, D. Lee, M. Klepal, C. Beder, M. Nikodem, S. Szymczak, P. Hoffmann, L. Selavo, D. Giustiniano, V. Lenders, M. Rea, A. Marcaletti, C. Laoudias, D. Zeinalipour-Yazti, Y.-K. Tsai, A. Bestmann, R. Reimann, L. Li, C. Zhao, S. Adler, S. Schmitt, V. Dentamaro, D. Colucci, P. Ambrosini, A. Ferraz, L. Martins, P. Bello, A. Alvino, V. Sark, G. Pirkl, and P. Hevesi, "A realistic evaluation and comparison of indoor location technologies: Experiences and lessons learned," in The 14th ACM/IEEE Conference on Information Processing in Sensor Networks (IPSN '15). "Association for Computing Machinery, April 2015. [Online]. Available: http://research.microsoft.com/apps/pubs/default.aspx?id=241 638

[5] M. Ficco, F. Palmieri, and A. Castiglione, "Hybrid indoor and outdoor location services for new generation mobile terminals," Personal and Ubiquitous Computing, vol. 18, no. 2, pp. 271-285, 2014. [Online]. Available: http://dx.doi.org/10.1007/s00779-013-0644-4

[6] C.-H. P. Chuan-Chin $\mathrm{Pu}$ and H.-J. Lee, Emerging Communications for Wireless Sensor Networks. InTech, February 2011, ch. Indoor Location Tracking Using Received Signal Strength Indicator, pp. 229-256. [Online]. Available: http://www.intechopen.com/books/emergingcommunications-for-wireless-sensornetworks/indoorlocation-tracking-using-received-signal-strength-indicator

[7] M. Najar and J. Vidal, "Kalman tracking based on tdoa for umts mobile location," 2001.

[8] L. Mainetti, L. Patrono, and I. Sergi, "A survey on indoor positioning systems," in proc. 22nd International Conference on Software, Telecommunications and Computer Networks, SoftCOM 2014, September 17-19, 2014, Split (Croatia), pp.111-120.

[9] Y. Gu, A. Lo, and I. Niemegeers, "A survey of indoor positioning systems for wireless personal networks," Communications Surveys Tutorials, IEEE, vol. 11, no. 1, pp. 13-32, First 2009.

[10] L. Mainetti, V. Mighali, L. Patrono, "A Location-aware Architecture for Heterogeneous Building Automation Systems, “ in 14th IFIP/IEEE Symposium on Integrated Network and Service Management, IM 2015, Ottawa (Canada), May 11-15, 2015.

[11] L. Mainetti, V. Mighali, and L. Patrono, "An IoT-based Usercentric Ecosystem for Heterogeneous Smart Home Environments," in Proc. 2015 IEEE International Conference on Communications, IEEE ICC 2015, London (UK), June 812, 2015.

[12] E. Times. (2012) Energy harvesting at the press of a button. [Online]. Available: http://www.eetimes.com/document.asp?doclsldo5(i)d=12801 56 pagelsldo5(n)umber $=1$

[13] E. GmbH, "Enocean at a glance."

[14] Kanan, R.; Elhassan, O., "Batteryless Radio System for Hospital Application," in 2016 SAI Computing Conference (SAI), 13-15 July, 2016

[15] Kanan, R.; Elhassan, O., "A Combined Batteryless Radio and $\mathrm{WiFi}$ Indoor Positioning System," ," in proc. 23nd International Conference on Software, Telecommunications and Computer Networks, SoftCOM 2015, September 17-19, 2015, Split (Croatia), pp.101-107.

[16] EnOcean. (2015) Radio technology. [Online]. Available: https://www.enocean.com/en/radio-technology/

[17] E. Alliance, "Enocean equipment profiles," 2014.

[18] S. Electric, "An004"lua enocean signal strength," 2013.

[19] E. GmbH, "Enocean gmbh."

[20] Q. Dong and W. Dargie, "Evaluation of the reliability of rssi for indoor localization," in Wireless Communications in Unusual and Confined Areas (ICWCUCA), 2012 International Conference on, Aug 2012, pp. 1-6.

[21] M. A. Ingram. Path loss. [Online]. Available: http://www.ece.gatech.edu/research/labs/sarl/tutorials/E CE4606/18-PathLoss.pdf

[22] S. Poykko and M. Spirito, "Method and system for estimating the position of a mobile device," Patent.

[23] N. Rao, X. Xu, and S. Sahni, "A computational geometry method for dtoa triangulation," in Information Fusion, 2007 10th International Conference on, July 2007, pp. 1-7. 
[24] C. Pu, "Development of a new collaborative ranging algorithm for rssi indoor location tracking in wsn," Ph.D. dissertation, PhD Thesis, Dongseo University, South Korea, 2009.

[25] C. Yang and H. rong Shao, "Wifi-based indoor positioning," Communications Magazine, IEEE, vol. 53, no. 3, pp. 150-157, March 2015.

[26] A. Papapostolou and H. Chaouchi, "Integrating rfid and wlan for indoor positioning and ip movement detection," Wireless Networks, vol. 18, no. 7, pp. 861-879, 2012. [Online]. Available: http://dx.doi.org/10.1007/s11276-012-0439-y

[27] F. Belloni, V. Ranki, A. Kainulainen, and A. Richter, "Anglebased indoor positioning system for open indoor environments," in Positioning, Navigation and Communication, 2009. WPNC 2009. 6th Workshop on, March 2009, pp. 261-265.

[28] X.-Y. Li, G. Calinescu, P.-J. Wan, and Y. Wang, "Localized delaunay triangulation with application in ad hoc wireless networks," Parallel and Distributed Systems, IEEE Transactions on, vol. 14, no. 10, pp. 1035-1047, Oct 2003.

[29] M. Abdalla, S. M. Feeney, and S. Salous, "Antenna Array and Quadrature Calibration for Angle of Arrival Estimation," SCI2003, Florida, 2003.

[30] Y.-S. Kuo, P. Pannuto, K.-J. Hsiao, and P. Dutta, "Luxapose: Indoor positioning with mobile phones and visible light," in Proceedings of the 20th Annual International Conference on Mobile Computing and Networking, ser. MobiCom '14. New York, NY, USA: ACM, 2014, pp. 447-458. [Online]. Available: http://doi.acm.org/10.1145/2639108.2639109

[31] P. Bahl and V. N. Padmanabhan, "Radar: An in-building rfbased user location and tracking system," in INFOCOM, 2000, pp. 775-784.

[32] C.-Y. Lin, W.-C. Peng, and Y.-C. Tseng, "Efficient in-network moving object tracking in wireless sensor networks," Mobile Computing, IEEE Transactions on, vol. 5, no. 8, pp. 10441056, Aug 2006.

[33] D. Giustiniano and S. Mangold, "Demo: Distance tracking using wlan time of flight," in Proceedings of the 9th International Conference on Mobile Systems, Applications, and Services, ser. MobiSys '11. New York, NY, USA: ACM, 2011, pp. 349-350. [Online]. Available: http://doi.acm.org/10.1145/1999995.2000029

[34] USB 300 / USB 300C / USB 300U (OEM) USB Gateway for EnOcean Radio.

[35] J. Liu, J. Reich, and F. Zhao, "Collaborative in-network processing for target tracking," EURASIP J. Appl. Signal Process, vol. 2003, pp. 378-391, Jan. 2003. [Online]. Available: http://dx.doi.org/10.1155/S111086570321204X

[36] N. Nakajima, "Indoor wireless network for person location identification and vital data collection," ISMICT'07, vol. TS9, December 2007.

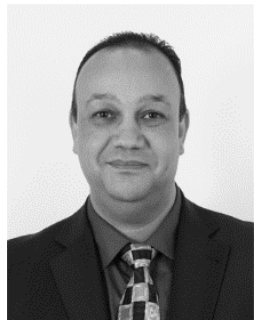

Riad Kanan received his Ph.D. in Electrical and Electronic Engineering from the Swiss Federal Institute of Technology (EPFL), Switzerland, in 1998. He started his academic career at the EPFL then he joined the industry in 2000. From 2000 to 2008 he was with EM Microelectronic-Marin Company (Swatch Group) and ON Semiconductor Company where he was involved in several state of the art $R \& D$ projects related to Low-Power Integrated Circuits. In 2008, he joined the University of Applied Sciences, Switzerland as a Professor. He was leading research projects between industry and academia and contributing to technology transfer to industry. He has invented and patented a technology for designing low-power indoor localization systems. Since 2013, he has been with the Department of Electrical and Computer Engineering, Abu Dhabi University, Abu Dhabi, United Arab Emirates. His research interests include low-power mixed-signal integrated circuits and systems, Radio Frequency Identification (RFID), wireless sensing systems, localization systems, energy efficient radios and emerging technologies. He is member of IEEE Solid-State Circuits, and IEEE Circuits and Systems Societies.

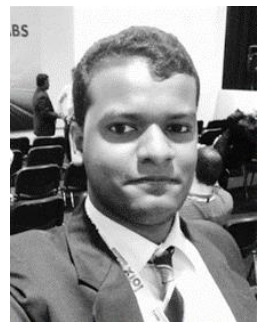

Obaidallah Elhassan has joined Abu Dhabi University, Abu Dhabi, United Arab Emirates in 2015 as Research Assistant. His research interests are mainly in Microelectronics and Internet of Things. He has conducted multiple researches and projects in these fields and, especially, in the area of Low power and selfpowered Wireless Sensor Networks for medical applications. 